\title{
$X$-ray cross-correlation analysis of liquid crystal membranes in the vicinity of the hexatic-smectic phase transition
}

\author{
R. P. Kurta, ${ }^{1}$ B. I. Ostrovskii, ${ }^{2}$ A. Singer, ${ }^{1, *}$ O. Y. Gorobtsov,${ }^{1,3}$ A. Shabalin, ${ }^{1}$ D. Dzhigaev, ${ }^{1,4}$ O. M. Yefanov, ${ }^{5}$ \\ A. V. Zozulya, ${ }^{1}$ M. Sprung, ${ }^{1}$ and I. A. Vartanyants ${ }^{1,4, \dagger}$ \\ ${ }^{1}$ Deutsches Elektronen-Synchrotron DESY, Notkestraße 85, D-22607 Hamburg, Germany \\ ${ }^{2}$ Institute of Crystallography, Russian Academy of Sciences, 119333 Moscow, Russia \\ ${ }^{3}$ National Research Center "Kurchatov Institute", Kurchatov Square 1, 123182 Moscow, Russia \\ ${ }^{4}$ National Research Nuclear University "MEPhI", 115409 Moscow, Russia \\ ${ }^{5}$ Center for Free-Electron Laser Science CFEL, Notkestraße 85, D-22607 Hamburg, Germany
}

(Received 14 August 2013; published 14 October 2013)

\begin{abstract}
We present an x-ray study of liquid crystal membranes in the vicinity of the hexatic-smectic phase transition by means of angular x-ray cross-correlation analysis. By applying two-point angular-intensity cross-correlation functions to the measured series of diffraction patterns the parameters of bond-orientational (BO) order in hexatic phase were directly determined. The temperature dependence of the positional correlation lengths was analyzed as well. The obtained correlation lengths show larger values for the higher-order Fourier components of BO order. These findings indicate a strong coupling between $\mathrm{BO}$ and positional order.
\end{abstract}

DOI: 10.1103/PhysRevE.88.044501

PACS number(s): 64.70.mj, 61.05.C-, 61.30.Gd

About 30 years ago it was realized that the phase transition between a two-dimensional (2D) crystal and the liquid phase can proceed through an intermediate hexatic phase [1]. The corresponding mechanism involves dissociation of dislocation pairs. The $2 \mathrm{D}$ hexatic phase is characterized by a sixfold quasilong-range bond-orientational (BO) order, while the positional order is short range and the shear modulus is zero. Phases with hexatic order have been found in several systems, such as electrons at the surface of helium [2], charged polymer colloids $[3,4]$, and smectic liquid crystals (LCs) [5-7]. In the last case a hexatic phase was experimentally observed quite unexpectedly in three-dimensional (3D) stacked molecular systems [8]. In the 3D hexatic phase the positional order is short range, while the $\mathrm{BO}$ order persists over a long range. However, the mere existence of a hexatic phase does not imply a specific melting mechanism, and the origin of hexatic order and its relation to the defect-mediated melting transition is still controversial. Smectic liquid crystals are particularly suitable to investigate these problems, as they can be suspended over an opening in a solid frame. Such smectic membranes are substrate-free and have a controlled thickness ranging from two to more than thousands of layers [9].

Smectic- $A$ membranes can be described as stacks of liquid layers. The in-plane structure is liquidlike with positional correlations between the molecules decaying exponentially with a correlation length $\xi_{0}$. While cooling a hexatic phase may occur, which shows a long-range BO order. This leads to a sixfold rotational symmetry and the $\mathrm{BO}$ correlations are characterized by the local ordering field $\psi(\mathbf{r}) \propto \exp [i 6 \theta(\mathbf{r})]$, where $\theta(\mathbf{r})$ is the angle between the "bonds" and some reference axis. Upon decreasing the temperature, the width of the radial-intensity peak decreases simultaneously with a further development of the BO order. This indicates a coupling

\footnotetext{
*Present address: The University of California, San Diego, La Jolla, CA 92093, USA.

†Corresponding author: ivan.vartaniants@ desy.de
}

between the positional correlations and the BO order [10]. Additionally, at even lower temperatures a 3D crystalline phase appears with a hexagonal in-plane lattice or a rectangular lattice with a so-called herringbone order [6,7].

The common way to study the $\mathrm{BO}$ order is to perform $\mathrm{X}$-ray or electron diffraction measurements on a single-domain hexatic film at different temperatures [11,12]. Information about the temperature evolution of the $\mathrm{BO}$ order parameters is typically obtained by fitting the measured azimuthal intensity distribution. We propose a different approach based on angular X-ray cross-correlation analysis (XCCA) [13-17] that allows determination of the $\mathrm{BO}$ order parameters directly, without fitting. Angular XCCA has been developed to study the structure of noncrystalline systems like liquids, amorphous systems, disordered ensembles of particles, etc. Smectic membranes are especially suited for Fourier analysis of intensity cross-correlation functions (CCFs). They are not influenced by substrate interactions and their two surfaces induce an almost perfect $2 \mathrm{D}$ alignment of the smectic layers. In this paper we aim to study a continuous crossover from the disordered smectic phase to hexatics, showing sixfold BO order, by applying the XCCA technique.

The in-plane structure factor in the smectic phase has the form of a broad ring due to short-range positional correlations between molecules. The presence of BO order in hexatic phases breaks the angular isotropy of the structure factor and leads to a sixfold modulation of the in-plane scattering. The angular Fourier decomposition of the intensity $I(q, \varphi)$ scattered at the momentum transfer vector $\mathbf{q}$ can be defined as

$$
I(q, \varphi)=I_{0}(q)+2 \sum_{n=1}^{\infty} I_{n}(q) \cos (n \varphi),
$$

where the radius $q=|\mathbf{q}|$ and azimuthal angle $\varphi$ are polar coordinates of $\mathbf{q}$, and $I_{n}(q)$ are the Fourier components of intensity $I(q, \varphi)$, with $I_{0}(q)$ representing the angular averaged intensity. In the case of a single hexatic domain, only the components $I_{n}(q)$ with $n=6 m, m=1,2,3,4, \ldots$, contribute to the expansion (1), while all other components have vanishing 
values [12]. The values of these Fourier components at $q=q_{0}$, corresponding to the maximum values of $I_{n}(q)$, are related to the BO order parameters [18] defined in Refs. [6,12].

Angular XCCA enables direct determination of the Fourier components $I_{n}(q)$ from the ensemble of diffraction patterns measured at different positions on the sample [14,15]. For each diffraction pattern a two-point cross-correlation function of the form

$$
C(q, \Delta)=\langle I(q, \varphi) I(q, \varphi+\Delta)\rangle_{\varphi}
$$

is defined, where $\Delta$ is the angular coordinate, and $\langle\cdots\rangle_{\varphi}$ denotes the angular average around a ring of radius $q$. The CCFs $C(q, \Delta)$ can be analyzed using a Fourier series decomposition $[14,15]$,

$$
C(q, \Delta)=C_{0}(q)+2 \sum_{n=1}^{\infty} C_{n}(q) \cos (n \Delta),
$$

where $C_{n}(q)$ are the Fourier components of the CCF, particularly $C_{0}(q) \equiv\langle I(q, \varphi)\rangle_{\varphi}^{2}$.

In the case of scattering from several domains, the values of the Fourier components $C_{n}(q)$ may fluctuate from position to position on the membrane [14-16]. However, averaging the Fourier components of the CCF $\left\langle C_{n}(q)\right\rangle_{M}$ over a sufficient number $M$ of diffraction patterns leads to an explicit relation $[15,16]$ between $\left\langle C_{n}(q)\right\rangle_{M}$ and $I_{n}(q),\left\langle C_{n}(q)\right\rangle_{M}=K\left|I_{n}(q)\right|^{2}$ [19]. Here $I_{n}(q)$ are the Fourier components of the angular expansion of intensity $I(q, \varphi)$ corresponding to a single domain, and $K$ is a scaling coefficient that depends on the number of domains contributing to diffraction patterns and the distribution of their orientations [15-17]. The XCCA approach can be directly used to determine the magnitudes of the Fourier components $\left|I_{n}(q)\right|$. For a single-domain case the scaling coefficient $K=1$ and $\left|I_{n}(q)\right|=\left\langle C_{n}(q)\right\rangle_{M}^{1 / 2}$.

The coherent $x$-ray scattering experiment [see Fig. 1(a)] was performed at the coherence beamline P10 of the PETRA III facility at DESY. The $x$ rays with the photon energy of $13 \mathrm{keV}$ were focused by compound refractive lenses (CRLs) $[20,21]$ to a spot about $3 \mu \mathrm{m}$ (FWHM) at the sample. A $2 \mathrm{D}$ detector (Pilatus $1 \mathrm{M}$ with $981 \times 1043$ pixels and a pixel size of $172 \times 172 \mu \mathrm{m}^{2}$ ) was positioned at $263 \mathrm{~mm}$ distance downstream from the sample. A specially designed sample stage FS1 together with a mK1000 temperature controller from INSTEC were used for preparation of LC thin films. Each film was aligned with its surface perpendicular to the direction of the incident beam.

Smectic membranes of the LC compound $n$-heptyl- $4^{\prime}-n$ pentyloxybiphenyl-4-carboxylate (75OBC) of different thickness in the range of 5-8 $\mu \mathrm{m}$ were drawn across a small circular glass aperture of $2 \mathrm{~mm}$ in diameter inside the chamber at $10-12{ }^{\circ} \mathrm{C}$ above the temperature of the smectic-hexatic phase transition. After stabilizing a film at elevated temperature $T$, it was gradually decreased with a temperature ramp $d T=0.3^{\circ} \mathrm{C} / \mathrm{min}$ to observe a sequence of LC phases [see Figs. 1(b)-1(e)]. At each temperature the sample was scanned in the plane perpendicular to the incident beam direction with a step size larger than the probe size. The exposure times were chosen in the range from $0.2 \mathrm{~s}$ up to $0.3 \mathrm{~s}$ per image to perform measurements in the nondestructive regime depending on the film thickness.
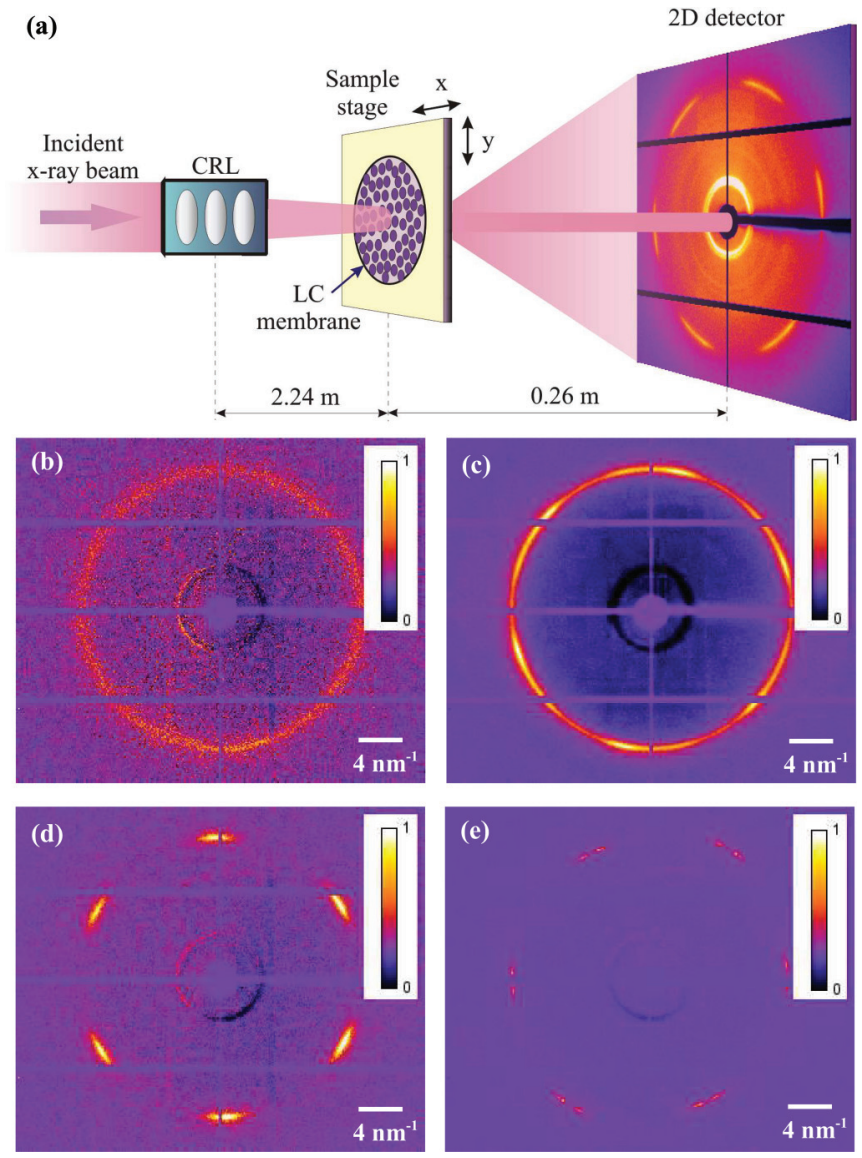

FIG. 1. (Color online) (a) Geometry of the scattering experiment showing the CRL transfocator, sample stage, and 2D detector. (b)-(e) Typical diffraction patterns measured for the LC membrane at different temperatures $T$. (b) Smectic phase $\left(T=64.25^{\circ} \mathrm{C}\right)$ with a scattering ring at $q_{0} \sim 14 \mathrm{~nm}^{-1}$. (c) Hexatic phase $\left(T=62.5^{\circ} \mathrm{C}\right)$ corresponding to scattering from few domains in different orientations. (d) Hexatic phase $\left(T=62.25^{\circ} \mathrm{C}\right)$ with a prominent sixfold symmetry typical for a single domain. (e) Crystalline phase $\left(T=58.75^{\circ} \mathrm{C}\right)$ with two domains of slightly different orientations.

Typical diffraction patterns corrected for background scattering and horizontal polarization of the synchrotron radiation corresponding to different phases appearing at distinct temperatures are shown in Figs. 1(b)-1(e). With gradual decrease of the temperature from $T=66$ to $58^{\circ} \mathrm{C}$ intensity distributions typical for smectic [Fig. 1(b)], hexatic [Figs. 1(c) and 1(d)], and low-temperature herringbone crystalline $E$ [22] [Fig. 1(e)] phases were measured. During different transverse scans in the $x-y$ plane [see Fig. 1(a)] we observed cases when the x-ray beam was scattered from a single domain [Fig. 1(d)], or from several domains [Figs. 1(c) and 1(e)].

In Fig. 2 the results of correlation analysis [Eqs. (2) and (3)] are presented for two different LC films. A large data set with $M=1024$ patterns was measured at the temperature $T=61.5^{\circ} \mathrm{C}$ for the first film containing several domains, and a small data set with $M=25$ diffraction patterns at $T=59.75^{\circ} \mathrm{C}$ for the second film with a single domain. The normalized averaged Fourier components $\left\langle C_{n}(q)\right\rangle_{M}$ calculated at $q_{0}=14.3 \mathrm{~nm}^{-1}$ for $1 \leqslant n \leqslant 40$ are presented in Figs. 2(a) and 2(b) for the several- and the single-domain case, 


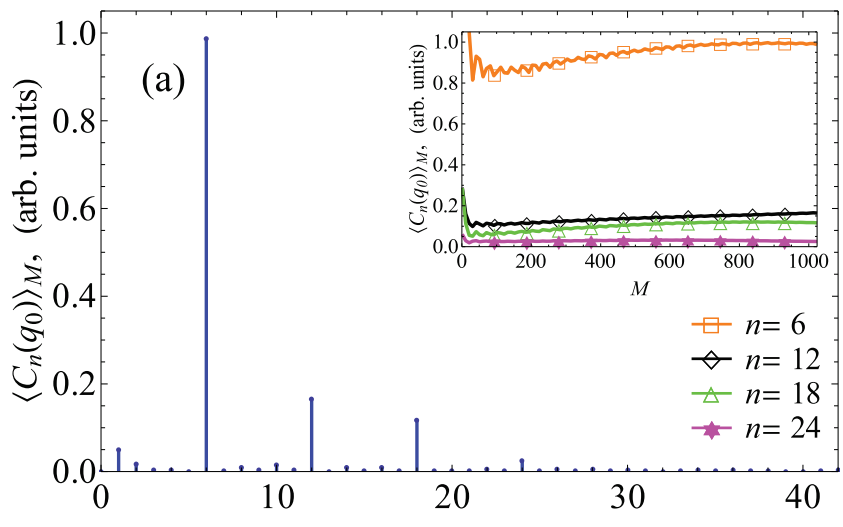

$n$
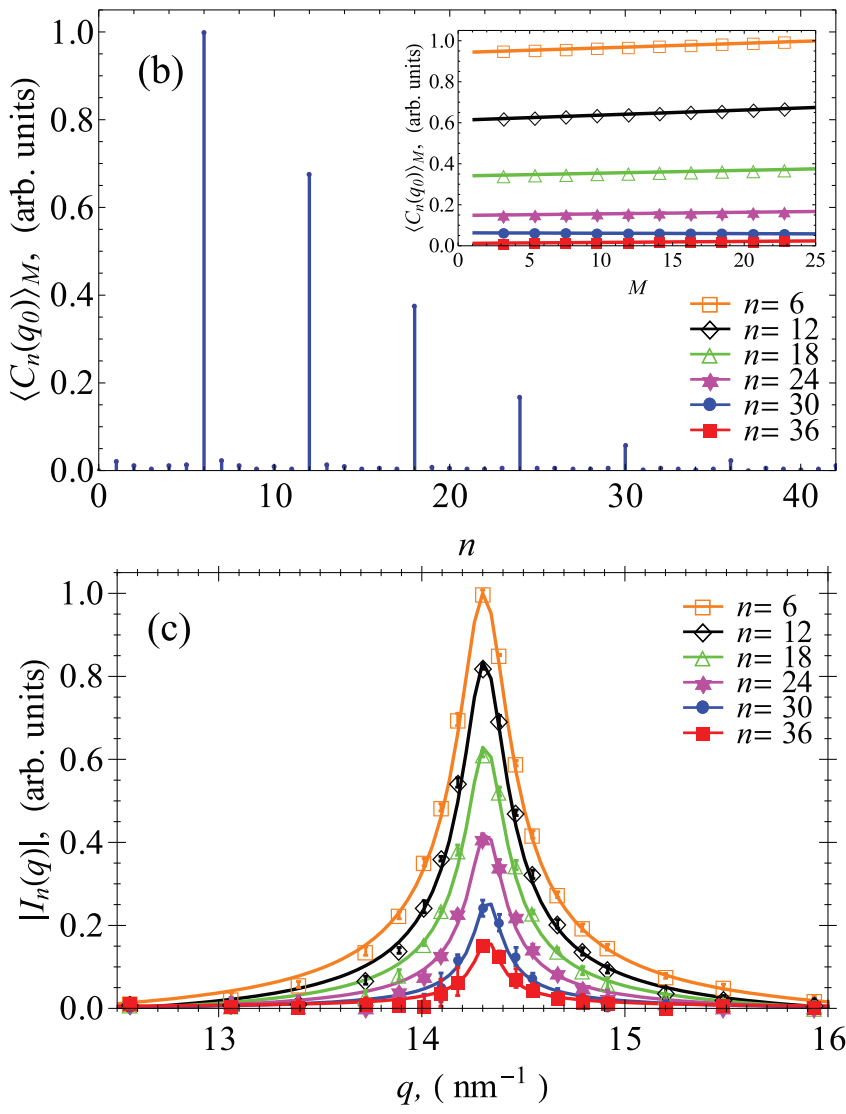

FIG. 2. (Color online) (a), (b) Normalized averaged Fourier components of CCFs $\left\langle C_{n}\left(q_{0}\right)\right\rangle_{M}$ determined at $q_{0}=14.3 \mathrm{~nm}^{-1}$ for $1 \leqslant n \leqslant 40$ for two different smectic membranes, corresponding to a several-domain case (a) and a single-domain case (b). Evolution of the dominant Fourier components as functions of $M$ is shown in the insets. (c) Normalized Fourier components $\left|I_{n}(q)\right|$ as functions of $q$ determined for the single-domain case. Solid lines are SRL fits to the experimental data (points). The Fourier components in (c) and in the insets are denoted as follows: $n=6$ (empty orange square), $n=12$ (empty black diamond), $n=18$ (empty green triangle), $n=24$ (filled magenta star), $n=30$ (filled blue circle), and $n=36$ (filled red square). The error bars in (c) are obtained by statistical analysis of five individual subensembles (five diffraction patterns each) from the whole set of $M=25$ patterns.

respectively. One can readily see that the dominant contribution to the spectrum is given by the Fourier components
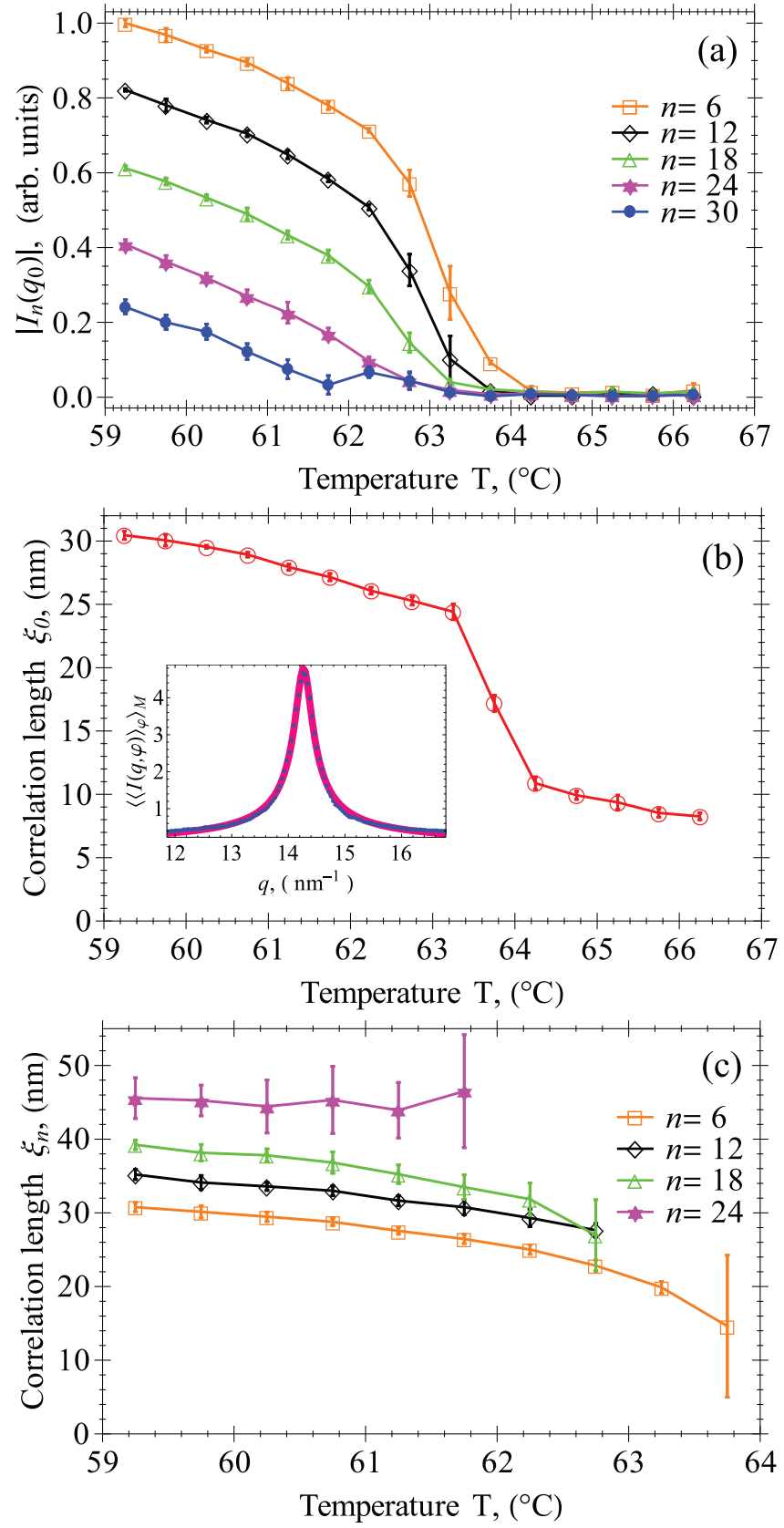

FIG. 3. (Color online) (a) Temperature dependence of BO order parameters $\left|I_{n}\left(q_{0}\right)\right|$ calculated for a single-domain case at $q_{0}=14.3 \mathrm{~nm}^{-1}$. (b) Temperature dependence of the correlation length $\xi_{0}(T)$, determined from the SRL fits of $\left|I_{0}(q)\right|$. A typical SRL fit for $\left|I_{0}(q)\right| \equiv\left\langle\langle I(q, \varphi)\rangle_{\varphi}\right\rangle_{M}$ is shown in the inset for $T=63.25^{\circ} \mathrm{C}$ (dots are experimental data, solid line is the SRL fit). (c) Temperature dependence of the correlation length $\xi_{n}(T)$ for $n=6,12,18$, and 24 obtained as in (b). The Fourier components here are denoted with the same line colors and markers as in Fig. 2.

with $n=6,12,18,24$, and $n=30,36$ in the single-domain case, whereas other Fourier components have vanishing values (except for $n=1$, which we attribute to a small misalignment of the detector). Evolution of the dominant Fourier components $\left\langle C_{n}(q)\right\rangle_{M}$ as functions of $M$ is shown in the insets of Figs. 2(a) and 2(b), demonstrating their statistical convergence. The normalized Fourier components determined for the 
single-domain case as $\left|I_{n}(q)\right|=\left\langle C_{n}(q)\right\rangle_{M}^{1 / 2}$ are presented as functions of $q$ in Fig. 2(c). We point out that the $q$ dependence of the Fourier components shown in Fig. 2(c) is a direct result of application of XCCA, which makes it advantageous as compared to fitting techniques.

We performed similar analyses at different temperatures and determined the temperature dependence of the $\mathrm{BO}$ order parameters $\left|I_{n}\left(q_{0}\right)\right|$ at $q_{0}=14.3 \mathrm{~nm}^{-1}$ for the single-domain case [see Fig. 3(a)]. It is readily seen that the hexatic order parameters decay as a power law upon approaching the hexatic-smectic transition, in good agreement with the multicritical scaling theory [23]. According to this theory [24], in the vicinity of the hexatic-smectic transition the BO order parameters measured for a single domain should follow a power law dependence as a function of $n$.

The shape of the diffuse radial peak $\left|I_{0}(q)\right|=\left\langle\langle I(q, \varphi)\rangle_{\varphi}\right\rangle_{M}$ determines the positional-order correlation length, and is described by a square-root Lorentzian (SRL) function $[6,10]$. We also fitted [see Fig. 2(c)] the higher-order Fourier components $\left|I_{n}(q)\right|$ by a SRL function $\left|I_{n}(q)\right|=B+S\left\{\gamma_{n} /\left[\left(q-q_{0}\right)^{2}+\right.\right.$ $\left.\left.\gamma_{n}^{2}\right]\right\}^{1 / 2}$, where $B$ is a background correction, $S$ is a scaling coefficient, $\gamma_{n}$ is the half width at half maximum (HWHM) of the corresponding Lorentzian function, and $q_{0}$ specifies the center of the function. The results of SRL fits demonstrate good agreement with the experimental data. For each Fourier component $\left|I_{n}(q)\right|$ we define the positional correlation length as $\xi_{n}=2 \pi /\left(\sqrt{3} \gamma_{n}\right)$, where $\sqrt{3} \gamma_{n}$ is the HWHM of the SRL function. For $n=0, \xi_{0}$ defines the conventional inplane positional correlation length $[8,9,12]$, while for $n>0$ the correlation lengths $\xi_{n}$ characterize the short-range positional correlations corresponding to higher harmonics of $\mathrm{BO}$ order.

We determined the temperature evolution of positional correlation lengths $\xi_{n}(T)$ for different $n$ from the corresponding SRL fits. The temperature dependence of $\xi_{0}(T)$ obtained for the single-domain case [see Fig. 3(b)] is in agreement with the previous results $[8,11,12,25,26]$. The correlation lengths $\xi_{n}(T)$ associated with the higher harmonics of $\mathrm{BO}$ order [see Fig. 3(c)] for each $n$ slightly decrease as the temperature grows, and the general character of decay is similar to that of $\xi_{0}(T)$. Remarkably, the correlation lengths $\xi_{n}(T)$ have larger values than $\xi_{0}(T)$, and consistently increase with the harmonic order $n$. This observation indicates that in hexatics the positional order is getting more extended in space if one takes the higher-order harmonics of BO order into consideration. This property is characteristic of well-developed BO order, where the contribution of the higher-order harmonics is larger.
In this situation coupling between the positional correlations and the BO order cannot be neglected. The evolution of the diffuse radial peak $\left|I_{0}(q)\right|$ in the vicinity of the hexaticsmectic transition in the presence of such a coupling has been studied by Aeppli and Bruinsma [10]. It was shown that upon decreasing temperature the width of the diffuse radial peak diminishes simultaneously with the development of the BO order. However, no specific dependence of the positional correlations on the $\mathrm{BO}$ order harmonics has been revealed. This question requires further theoretical and experimental analysis.

In summary, we applied XCCA to determine the BO order parameters in the hexatic phase close to the hexatic-smectic phase transition. Fourier analysis of the two-point CCFs provided direct access to the parameters of BO and their temperature dependence in the hexatic phase. The $\mathrm{BO}$ parameters of orders $n=6,12,18,24,30$, and 36 were determined. From analysis of the radial-intensity profiles we determined the temperature dependence of the in-plane positional correlation length in hexatic membranes. Moreover, the application of XCCA allows us to measure the correlation lengths $\xi_{n}(T)$ for $n>0$, associated with higher harmonics of BO order in the hexatic phase. The corresponding correlation lengths $\xi_{n}(T)$ consistently show larger values for the higher-order Fourier components that is related to the interplay between positional and $\mathrm{BO}$ order. Our results demonstrate the ability of XCCA to directly measure the parameters of $\mathrm{BO}$ order in smectic membranes. This makes it a relevant tool for studying angular correlations and other order parameters in various partially ordered molecular ensembles. We expect that our results will stimulate further theoretical studies of ordering phenomena in hexatics and other LCs, and development of experimental techniques that enable quantitative investigation of ordering in molecular films composed of multiple domains.

We thank Ewa Gorecka, C. C. Huang, and Wim de Jeu for providing us hexatic materials and for stimulating discussions, M. Altarelli and J. Brock for fruitful discussions, and S. Funari for a careful reading of the paper. We are grateful to Sergey Sulyanov and Pavel Dorovatovskii for preliminary characterization of hexatic liquid crystals at Kurchatov Synchrotron Center, Moscow. Part of this work was supported by BMBF Proposal No. 05K10CHG "Coherent Diffraction Imaging and Scattering of Ultrashort Coherent Pulses with Matter" in the framework of the German-Russian Collaboration "Development and Use of Accelerator-Based Photon Sources" and the Virtual Institute VH-VI-403 of the Helmholtz Association.
[1] B. I. Halperin and D. R. Nelson, Phys. Rev. Lett. 41, 121 (1978).

[2] D. C. Glattli, E. Y. Andrei, and F. I. B. Williams, Phys. Rev. Lett. 60, 420 (1988).

[3] C. A. Murray and D. H. Van Winkle, Phys. Rev. Lett. 58, 1200 (1987).

[4] R. E. Kusner, J. A. Mann, J. Kerins, and A. J. Dahm, Phys. Rev. Lett. 73, 3113 (1994).

[5] K. Strandburg, Bond-Orientational Order in Condensed Systems (Springer, New York, 1992).
[6] J. D. Brock, R. J. Birgenaeau, J. D. Litster, and A. Aharony, Contemp. Phys. 30, 321 (1989).

[7] T. Stoebe and C. C. Huang, Int. J. Mod. Phys. B 9, 2285 (1995).

[8] R. Pindak, D. E. Moncton, S. C. Davey, and J. W. Goodby, Phys. Rev. Lett. 46, 1135 (1981).

[9] W. H. de Jeu, B. I. Ostrovskii, and A. N. Shalaginov, Rev. Mod. Phys. 75, 181 (2003).

[10] G. Aeppli and R. Bruinsma, Phys. Rev. Lett. 53, 2133 (1984). 
[11] C. F. Chou, J. T. Ho, and S. W. Hui, Phys. Rev. E 56, 592 (1997).

[12] J. D. Brock, A. Aharony, R. J. Birgeneau, K. W. Evans-Lutterodt, J. D. Litster, P. M. Horn, G. B. Stephenson, and A. R. Tajbakhsh, Phys. Rev. Lett. 57, 98 (1986).

[13] P. Wochner, C. Gutt, T. Autenrieth, T. Demmer, V. Bugaev, A. Diaz-Ortiz, A. Duri, F. Zontone, G. Grübel, and H. Dosch, Proc. Natl. Acad. Sci. USA 106, 11511 (2009).

[14] M. Altarelli, R. P. Kurta, and I. A. Vartanyants, Phys. Rev. B 82, 104207 (2010); 86, 179904(E) (2012).

[15] R. P. Kurta, M. Altarelli, E. Weckert, and I. A. Vartanyants, Phys. Rev. B 85, 184204 (2012).

[16] R. P. Kurta, R. Dronyak, M. Altarelli, E. Weckert, and I. A. Vartanyants, New J. Phys. 15, 013059 (2013).

[17] R. P. Kurta, M. Altarelli, and I. A. Vartanyants, Adv. Condens. Matter Phys. 2013, 959835 (2013).

[18] The following decomposition of the azimuthal intensity was used previously to describe BO order in LCs [6,12]: $I(\varphi)=I_{0}[1 / 2+$ $\left.\sum_{m=1}^{\infty} C_{6 m} \cos (6 m \varphi)\right]$. Here, $I_{0}$ and the BO order parameters $C_{6 m}$ are related to the Fourier components $I_{n}(q)$ defined in Eq. (1) as $I_{0}=2 I_{0}\left(q_{0}\right)$ and $C_{6 m}=I_{n}\left(q_{0}\right) / I_{0}\left(q_{0}\right), n=6 m$, with $q_{0}$ corresponding to the maximum of $I_{n}(q)$.
[19] The interference terms from different domains were neglected in derivation of this equation.

[20] A. V. Zozulya, S. Bondarenko, A. Schavkan, F. Westermeier, G. Grübel, and M. Sprung, Opt. Express 20, 18967 (2012).

[21] A. Singer and I. A. Vartanyants, J. Synchrotron Radiat. 21 (2014) [in print].

[22] R. Geer, T. Stoebe, C. C. Huang, R. Pindak, G. Srajer, J. W. Goodby, M. Cheng, J. T. Ho, and S. W. Hui, Phys. Rev. Lett. 66, 1322 (1991).

[23] A. Aharony, R. J. Birgeneau, J. D. Brock, and J. D. Litster, Phys. Rev. Lett. 57, 1012 (1986).

[24] The multicritical scaling theory [23] predicts a power law decay of the BO order parameters $C_{6 m}=I_{n}\left(q_{0}\right) / I_{0}\left(q_{0}\right), n=6 m$, for a single-domain system. According to this theory, $C_{6 m}=$ $C_{6}(T)^{\sigma_{m}}, \sigma_{m}=m+\lambda m(m-1)$, and $\lambda \approx 0.3$ in the $3 \mathrm{D}$ case. We observed the same power law with $\lambda \approx 0.3$ for the single-domain case, but our results for the several-domain case do not follow this power law and require further analysis.

[25] S. C. Davey, J. Budai, J. W. Goodby, R. Pindak, and D. E. Moncton, Phys. Rev. Lett. 53, 2129 (1984).

[26] W. H. de Jeu, A. Fera, O. Konovalov, and B. I. Ostrovskii, Phys. Rev. E 67, 020701 (2003). 